\title{
Irréductibles défenseurs de la composition improvisée à New York
}

\section{Amandine Pras}

Cet article propose une élaboration du concept de composition improvisée basée sur les citations recueillies lors d'entretiens individuels autour de la pratique d'improvisation de 12 musiciens professionnels vivant à New York, ${ }^{1}$ soient Michaël Attias (saxophones alto et baryton), Jim Black (batterie et électronique), Todd Capp (batterie), Daniel Carter (saxophones soprano, alto et ténor, flûte traversière, clarinette et trompette), Mary Halvorson (guitare), John Hébert (contrebasse), Ingrid Laubrock (saxophones soprano et ténor), Tony Malaby (saxophones soprano et ténor), Tom Rainey (batterie), Angelica Sanchez (piano), Matthew Shipp (piano), et Satoshi Takeishi (batterie, percussions et électronique). Au début de l'enquête, nous utilisions les termes free jazz et improvisation libre pour présenter notre projet aux musiciens, termes qui à première vue correspondaient à la pratique musicale observée. Cependant, comme on le verra en détail au cours de cet article, ces termes ont été remis en question par les musiciens interrogés. Lorsque l'auteure a demandé à Michaël Attias et Jim Black quel genre musical les définissait le mieux pour qu'elle sache comment les présenter lors de concerts en Inde, ils lui ont répondu le jazz (nous invitons le lecteur à visionner une improvisation en duo de Michael Attias et Jim Black à Calcutta ci-dessous). En effet, si les improvisations de ces musiciens new-yorkais sonnent parfois très éloignées de ce qu'on entend communément par jazz, ils s'identifient à ce que le jazz représente au niveau du mode de vie et de l'approche créative. Résumons ici cette identification par des concepts largement discutés dans la littérature et qui caractérisent la pratique du jazz à travers son histoire, soit l'utilisation sans cesse renouvelée de l'improvisation (Berliner, 1994), la recherche constante de plaisir (Roueff, 2013), de liberté (Borgo, 2002), ou encore de transcender les esthétiques du passé et les états d'être à travers l'expression musicale individuelle ou collective (McCormack, 2012).

Cliquez sur l'image pour visionner la video:

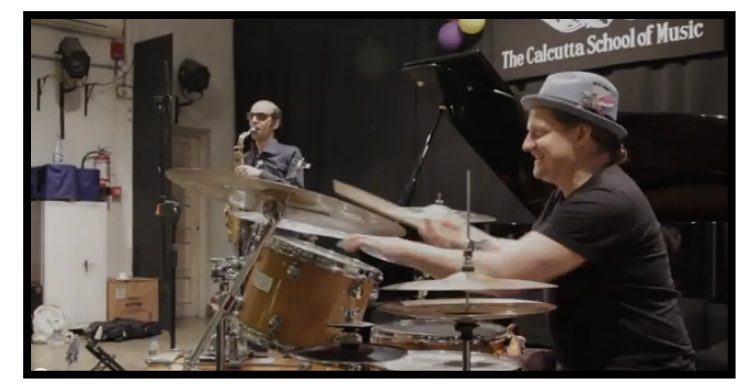

Michaël Attias et Jim Black en duo, séance d'ouverture d'un atelier jazz à la Calcutta School of Music le 11 janvier 2015.

Nous avons identifié les concepts de composition improvisée, instantanée, spontanée, ou encore instinctive des données verbales de ces musiciens lorsqu'ils décrivent leur approche de création qu'ils comparent, sans l'opposer, à l'interprétation d'une composition ou à l'improvisation à partir de contraintes structurelles et/ou musicales préétablies. Dans la littérature, cette approche de création musicale s'intègre dans le concept d'improvisation non idiomatique selon Bailey (1993), aussi appelée improvisation libre et définie en opposition à la composition du point de vue de la notion d'œuvre d'art (Canonne, 2012, p.4). Les musiciens de l'enquête réfutent cette définition car ils visent à créer une composition en temps réel via l'improvisation comme processus créatif. Nous allons détailler au cours de l'article leurs différentes approches pour accéder à un état d'improvisation qui permet d'aboutir à une composition.

Certains des musiciens ont spontanément mentionné les enregistrement qui les ont exposés en premier lieu à cette approche de création musicale, soient les albums Free Jazz: A Collective Improvisation (Atlantic, 1961) du double quartet d'Ornette Coleman ; Fire Music (Impulse !, 1965) d'Archie Shepp dédié à Malcom X ; Live ! at the Village Vanguard et Meditations (Impulse !, 1962, 1966) du quartet classique de John Coltrane ; et Miles Smiles (Columbia, 1967) du $2^{\text {nd }}$ quintet de Miles Davis. Ces références, parfois incomprises au premier abord mais leur suscitant un intérêt particulier, ou tout de suite décisives dans leur choix d'orientation stylistique, nous montrent qu'ils ont puisé leur inspiration artistique dans le jazz africain-américain des années 1960 communément appelé free jazz.

Toujours dans la littérature, l'improvisation libre se distingue du free jazz pour discerner deux origines culturelles différentes à une pratique similaire datant dans les deux cas des années 1960 et toutes deux intrinsèquement liées aux évènements politiques occidentaux de cette période (Drott, 2001). Lewis (1996) qualifient ces deux traditions de l'improvisation comme eurological et afrological, la première prenant sa source dans la tradition de la musique contemporaine européenne qui inclut des compositeurs tels que John Cage (Saladin, 2015), la seconde amenant des musiciens essentiellement issus de la communauté africaine-américaine à réagir contre l'évolution commerciale du jazz (Carles \& Comolli 1971, p.391-393), résistance dans la lignée de la tradition du blues (Baraka dit LeRoi Jones, 
1997). Macdonald et Wilson (2005, p.396) mentionnent également l'association à la pratique intérieure pour la tradition eurological versus une philosophie basée sur l'expérience partagée pour la tradition afrological. Par sa situation géographique et son histoire, New York a fourni un terrain de développement privilégié pour ces deux traditions musicales avant-gardistes pendant la seconde moitié du $20^{\text {ème }}$ siècle. Aujourd'hui, alors que les origines culturelles dont elles sont issues jouent encore un rôle majeur dans la définition identitaire de certains musiciens de la scène jazz new-yorkaise (dont certains participants de l'enquête), leurs caractéristiques au niveau des approches de création musicale tendent à s'entremêler dans une communauté artistique qui encourage les musiciens issus de divers horizons, parfois au-delà des frontières occidentales, à improviser ensemble sur la même scène.

Les 12 musiciens de l'enquête ont été choisis selon des critères d'accomplissement professionnel, soit plus de 15 ans d'expérience internationale en tant qu'improvisateurs reconnus, et une voix artistique unique et puissante. L'auteure les a également sélectionnés pour représenter, autant que possible, la diversité culturelle et générationnelle de cette communauté. Par ailleurs, ils se connaissent entre eux et sont susceptibles de jouer ensemble ; ils se produisent dans des lieux alternatifs tels qu'iBeam, Barbès ou Roulette à Brooklyn, Cornélia Street Café ou le Stone à Manhattan. Ils jouaient régulièrement à la Knitting Factory et au Tonic avant leur fermeture ; ils sont donc désignés comme faisant partie de la scène Downtown New York, catégorisation qu'ils n'acceptent pas tous car elle inclut des approches artistiques qui diffèrent de la leur. Pour Tony Malaby, Angelica Sanchez, Tom Rainey ou encore John Hébert, leur arrivée à New York a joué un rôle instigateur dans leur approche de la composition improvisée qu'ils ont développée en jouant avec des musiciens locaux de la génération précédente. Nous nous situons donc bien dans la tradition du jazz au niveau du transfert des connaissances, même si certains musiciens de l'enquête ont fait leurs premiers pas d'improvisateurs avec une formation poussée en musique classique et contemporaine, ou en jouant des reprises de pop-rock et de funk, ou encore des musiques latines.

Les entretiens dont il est question dans cet article marquent le début d'un projet collaboratif de recherche et création initié par l'auteure qui travaille en tant que réalisatrice d'enregistrements au sein de cette scène musicale. Ils ont été menés, transcris ${ }^{2}$ et analysés par l'auteure; cependant, pour minimiser le biais introduit par la formulation des questions et recueillir des descriptions individuelles au-delà des représentations consensuelles de la pratique (Delepaut, 2009), le guide d'entretiens a été développé en collaboration entre l'auteure et la linguiste Caroline Cance, spécialisée dans l'analyse des pratiques humaines et de l'expérience sentie. Cet article se concentre sur trois thèmes identifiés lors de l'analyse des descriptions verbales des musiciens selon la méthode de comparaison constante de la théorie ancrée (Grounded Theory, Corbin \& Strauss, 2008): 1) leur définition de la composition improvisée en comparaison avec d'autres contextes de création musicale; 2) leurs procédés créateurs incluant leur pratique quotidienne et leurs stratégies pour favoriser l'expression artistique à travers l'improvisation; et 3) les liens entre leur approche artistique et leur philosophie de vie. Les musiciens de l'enquête ont eu le choix d'associer ou non leur nom à toutes leurs citations ou à une partie de leurs citations sélectionnées par l'auteure.

\section{Définitions de la composition improvisée par les musiciens de l'enquête}

Comme introduit précédemment, à la question quand avez-vous commencé à improviser librement?, les musiciens ont argumenté à propos du concept d'improvisation libre: « Je ne pense pas qu'il y ait d'un côté l'improvisation libre et de l'autre des compos, des morceaux pop, des standards. [. . .] Je ne fais pas vraiment cette distinction, [. . .] en fait, j'aspire à créer quelque chose qui est complètement accompli, que ce soit une composition, une symphonie, un quatuor à cordes, ou quoique ce soit d'autre." Jim Black renforce cette position par la notion de responsabilité artistique qui serait associée à un procédé compositionnel et non à l'improvisation libre: « On prend des décisions même si elles viennent peut-être d'un endroit très honnête de l'intérieur, un endroit très spontané au cœur de notre être. Mais tu prends tout de même des décisions, si bien que l'improvisation libre, ça n'existe pas, ce n'est que de la composition spontanée. On compose des choses et on est responsable de chaque son qu'on produit." Cependant, après une discussion sur la terminologie, ils nous ont fait part de leurs premières expériences d'improvisation dès leur plus jeune âge sans savoir de quoi il s'agissait, soit une découverte naturelle de la musique et de leur instrument. Plusieurs ont précisé qu'entre l'enfance et la redécouverte de cette pratique à l'âge adulte, souvent provoquée par des rencontres de musiciens à New York (ou à Londres pour Ingrid Laubrock), il s'était passé plus de vingt ans. Un des musiciens explique que l'exploration via l'improvisation libre, en plus d'être libératrice, a eu un impact sur sa façon de jouer dans d'autres situations musicales : "Je crois qu'une fois que j'ai commencé à faire ça, je me suis dit waouh ! [ . . ] En plus il y a quelque chose d'autre de libérateur là-dedans et ça m'aide à dialoguer avec cet autre côté de moi-même." Satoshi Takeishi et Jim Black, deux anciens étudiants de l'école de musique de Berkeley ont aussi explicité leur besoin d'improviser hors style pendant leurs études pour contrebalancer un enseignement traditionnel qui mettait l'accent sur le bebop, le big band, le funk et les musiques latines.

Bien que nous ayons évité d'utiliser le terme free jazz dans les questions du guide d'entretien, Todd Capp et Matthew Shipp ont spontanément élaboré sur ce genre musical. À propos de son retour sur scène dans les années 2000 après des expériences musicales diverses entre les années 1960 et 1980, suivies de l'arrêt complet de ses activités 
musicales pendant une dizaine d'années, Todd Capp témoigne : « C'était vraiment super comme sensation, et une superbe expérience, ce que je veux dire c'est que ça peut marcher, le free jazz n'est pas forcément cette musique forte et agressive qui [. . .] lui a collé cette mauvaise réputation." Plus tard dans l'entretien, il rapporte : "Quand je rencontrais Daniel Carter dans la rue on se mettait à parler, et avec lui je retrouvais l'espoir que le free jazz n'était pas qu'une musique forte agressive égocentrique et macho. ${ }^{7}$ " Ces citations suggèrent que le genre musical free jazz s'est transformé au cours du temps, tout du moins à New York. D'après Matthew Shipp, il s'agit bien d'un genre et non d'improvisation non-idiomatique :

Si tu es au courant de l'existence de cette forme particulière d'expression qu'est le free jazz et si tu veux en faire partie, la première chose qui te vient à l'esprit c'est de fabriquer des trucs sur le vif, et ça c'est avec l'improvisation que ça marche. Même si tu imagines ça dans le contexte du bebop ou de n'importe quoi d'autre, l'improvisateur sera toujours celui qui se pointe devant son public en temps réel, et élabore sa musique en se concentrant sur la dimension spatio-temporelle. ${ }^{8}$

Plus tard dans l'entretien :

Dans le free, si tu en écoutes suffisamment, il y a un nombre d'habitudes, d'accord t'es bel et bien assis en train d'inventer des trucs, mais il y a comment dirais-je, je ne veux pas appeler ça des clichés, mais il y a des façons de s'y prendre que ceux qui jouent en ville ont vite pigées. Je ne veux pas dire des mélodies bien précises ou des harmonies ou des rythmes, mais il y a certaines façons de se débrouiller avec l'activité en groupe ou en solo. Et si tu écoutes assez de free jazz des années 60 tu dois pouvoir vite choper quelques uns de ces trucs. Donc ça te donne un cadre, qu'il s'agisse de motifs de type question réponses, ou une façon de jouer modal à la post-Coltrane, ou genre bop free à la post-Ornette, tu vois il y a quelque trucs gestuels que les musiciens captent de façon assez rigoureuse, et t'es tout le temps en train de réagir à des prétextes. ${ }^{9}$

Ces gestes musicaux explicités par Matthew Shipp caractérisent le genre free jazz des années 1960, gestes que l'on retrouve souvent dans les compositions improvisées des musiciens de l'enquête. Néanmoins, la plupart ne veulent pas êtres associés à la scène dite free jazz.

Tous ces musiciens sont aussi impliqués dans différents genres musicaux qui incluent le rock, les standards ou les compositions originales de jazz, la musique contemporaine, le hip-hop, le punk et le noise. Une des questions du guide d'entretien traitait de la différence entre l'expérience d'improviser librement et de jouer dans d'autres contextes de création musicale. Pour certains, le genre musical n'influence par leur approche artistique, citons Angelica Sanchez :

Toute cette idée de jouer free est un peu bizarre pour moi parce que je ne pense pas qu'il y ait de différence entre les gars qui jouent du be-bop ou n'importe quelle autre musique d'ailleurs, et jouer free. Je te l'accorde, le langage n'est pas exactement le même, à l'écoute, ce n'est pas la même chose. [. . .] Peut-être que c'est quelque chose qu'on reconnaît moins, mais si on regarde seulement la façon dont la musique est fabriquée, et les informations et les ingrédients dont tu as besoin pour faire cette musique, c'est la même chose. Mais ça m'a pris vingt ans pour m'en rendre compte. $^{10}$

Daniel Carter affirme même qu'il peut y avoir plus de liberté dans une situation d'interprétation que dans le free jazz, et il prend pour exemple sa propre expérience de reprises de chansons populaires avec ses amis à l'adolescence. Cependant, Daniel Carter comme Mary Halvorson et Todd Capp précisent qu'ils apprécient les situations où ils sont libres de faire ce qu'ils veulent. Ingrid Laubrock rapporte également qu'elle se sent plus libre de s'exprimer musicalement sans contrainte formelle préétablie :

Des fois, quand je jouais de la musique cadrée par une forme, disons, comme un standard, ou n'importe quoi qui est écrit à partir d'une forme, j'avais l'impression qu'on ne s'attaquait pas assez à la "grande forme", juste à cause du fait qu'il y avait des arrangements, on était obligé d'aller vers un certain point, on n'allait pas où je sentais que la musique pouvait aussi aller. ${ }^{11}$

Pour contourner cette contrainte structurelle, plusieurs d'entre eux sont susceptibles d'écrire une mélodie ou un enchaînement harmonique qu'ils proposent à leur ensemble sans imposer aucune forme, y compris le démarrage de ces éléments qu'ils laissent libre au flot de l'improvisation. Tony Malaby affirme d'ailleurs que ces éléments musicaux, s'ils sont inspirant, lui permettent de se renouveler: " J'ai l'impression que si je ne faisais qu'improviser, je pourrais m'enfermer dans une certain type de routine comme j'ai pu en entendre. " "Pour ses solos de percussions et électronique, Satoshi Takeishi utilise le mot composition au sens littéral, donc pour signifier un assemblage de 
timbres, d'interfaces et d'effets. Une fois l'assemblage fixé, il improvise avec ces différents éléments pour se familiariser avec cette composition avant de la jouer en public (nous invitons le lecteur à visionner une de ces compositions filmée lors d'une séance de studio à l'université de New York).

Cliquez sur l'image pour visionner la video:

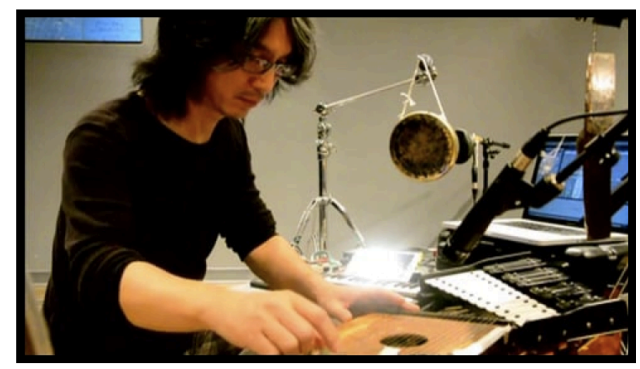

Enregistré au Studio James Dolan, New York University, le 11 mai 2014.

\section{Procédés créateurs de composition improvisée : plusieurs approches}

Les musiciens de l'enquête ont tous largement insisté sur l'importance d'être dans le moment présent comme élément indispensable à la composition improvisée. Ce consensus au sein de la population observée a été exprimé par toutes sortes de descriptifs, soit "focalisé, centré, concentré, engagé, etc. " Jim Black a utilisé la métaphore : " Clic, j'allume la lumière. ${ }^{13}$ " pour illustrer le moment où il entre dans l'improvisation. Lorsque l'un des musiciens a décrit des expériences d'improvisation intenses par: "J'ai la sensation que tout m'est dicté ${ }^{14}$ ", l'auteure lui a demandé si cette dictée était musicale ou sous forme de mots, et il a répondu:

Ouais, c'est musical, c'est des gestes. Ouais. Je suis là, à entendre et je suis complètement dans cet ici et maintenant, si présent, tellement dans l'écoute et dans la conscience de cette écoute, et je suis si connecté aux autres [. . .]. Si je suis tout seul je suis complètement connecté au public et à l'espace et aux instruments. Si je joue avec d'autres nous sommes aussi connectés entre nous. Ce qui ne veut pas dire que nous écoutons tous la même chose, mais nous sommes tous si connectés que les morceaux se déroulent d'eux-mêmes. Je cherche juste à ne pas rester sur le chemin tracé, c'est là que je me sens le mieux, et pour moi, c'est quand j'ai ce sentiment-là que c'est l'impro la plus libre. Parce que ça n'a pas été conditionné par aucune volonté extérieure au morceau. Et aussi, c'est la première fois que ce morceau se joue. ${ }^{15}$

Cette réponse complète résume une sorte d'état d'improvisation décrit par plusieurs des musiciens de l'enquête soit une écoute profonde qui leur permet d'êtres connectés. Pour ce musicien, cette connexion est essentiellement extérieure, soit avec le public, l'espace, l'instrument et les autres musiciens. Pour d'autres, il s'agit d'une connexion intérieure, ou encore transcendantale qu'ils illustrent par l'idée d'entrer dans une zone ou dans un tout pendant le moment d'improvisation.

Pour expliciter le procédé créateur de ces musiciens, l'auteure a souvent été amenée à leur demander s'ils pensaient consciemment ou non pendant l'improvisation. Les réponses diffèrent beaucoup selon les musiciens. Daniel Carter pense souvent à une référence tonale ou modale, même si celle-ci évolue très rapidement au cours de la composition improvisée. Ingrid Laubrock pense également en termes musicaux et analyse parfois ce qu'il se passe. Au contraire il y a ceux qui évitent de penser, citons par exemple Angelica Sanchez : «Ce que j'essaye de faire quand j'improvise, au moment où je m'assieds pour jouer toute seule, ou avec d'autres musiciens, c'est de ne pas penser. Le but est de se tenir à l'écart, et de s'en tenir à canaliser la moindre chose qui arrive. ${ }^{16}$ „ L'idée de canaliser illustre d'ailleurs la connexion transcendantale mentionnée précédemment. II y a aussi ceux qui décrivent leur procédé d'improvisation comme une réaction instinctive plutôt que des pensées, citons Matthew Shipp : "Je ne sais pas ce que ça signifie de penser, t'essayes vraiment de faire en sorte que tout se passe au niveau de l'instinct, et t'essayes de capter les informations tout en réagissant instinctivement et dans l'instantanéité de cette réaction, il ne s'agit pas vraiment de penser, c'est réagir. ${ }^{17}$ " Jim Black explique également qu'il fait confiance à son corps pour réagir naturellement mais il n'exclue pas le fait de penser ce qu'il décrit comme des milliers de décisions à prendre. Finalement, plusieurs ont explicité une dualité entre deux états, illustrée par cette métaphore du mixeur : " II y a une partie de toi qui agit un peu comme un mixeur, tu as tous ces différents boutons. Donc il y en a un qui joue, et qui fait vraiment que jouer tu vois, penser, rêver et qui lâche tout. Et l'autre, lui, a des boutons pour [. . .] l'activité cérébrale, ou gestuelle, physique le système nerveux, le cœur, la mémoire. ${ }^{18}$ " Tony Malaby détaille aussi cette dualité qu'il n'expérience que dans le contexte de composition improvisée : 
Ce sentiment est si primitif au niveau de comment la création agit, et c'est si intuitif et basique. [... .] Et en même temps que tout ça se passe, il y a des stratégies très évoluées, et on pense, on pense en terme de composition, et on fait des exercices de corde raide chorégraphiques très intelligents, et on cherche à doser les deux. Et le fait d'avoir ces deux activités qui oscillent vraiment à haute fréquence, je ne retrouve pas ça quand je joue d'autres types de musique. ${ }^{19}$

Cette citation introduit ce que Tony Malaby nomme son thought process soit une pratique intense de l'instrument pour cataloguer des sonorités et des techniques de jeu auxquelles il associe des moments de vie, des sensations, des séquences de films qu'ils nomment visualisations. Pendant l'improvisation, il utilise ces visualisations pour faire le lien entre la musique des autres musiciens et les sons qu'il produit. Chaque composition improvisée propose un nouveau scénario de ces visualisations. Dans une idée un peu semblable, Satoshi Takeishi voit des images, plus précisément des formes et des couleurs qu'il explore lors de sa pratique avec un assemblage d'instruments fixe qu'il nomme composition. Ces procédés créateurs sont vraiment propres à Tony Malaby et Satoshi Takeishi. Cependant, d'autres musiciens de l'enquête ont aussi détaillé leur pratique quotidienne basée soit sur des exercices qu'ils ont eux-mêmes développés soit à travers des moments d'improvisation en solitaire dans le but d'augmenter leur vocabulaire et de trouver de nouvelles idées compositionnelles. Ils ont mentionné une évolution entre leur pratique de jeunesse lorsqu'ils élaboraient leur langage qui consistait à répéter des exercices techniques, jouer avec des concepts d'improvisation, travailler sur des transcriptions d'improvisations ou improviser directement avec les enregistrements, et leur pratique quotidienne actuelle qui est d'ailleurs devenue complètement inutile pour certains d'entre eux. Plusieurs musiciens, dont Tony Malaby, ont aussi expliqué comment la pratique de l'instrument les rend plus libres car elle les aide à résister aux pensées susceptibles de détruire le moment d'improvisation, soit des pensées de critique ou de jugement intérieurs, ou encore des pensées liées au stress de la vie courante. Ces pensées parasites les forcent à s'arrêter de jouer au milieu d'une composition improvisée et elles auraient moins de chance de percer lorsque le musicien se sent vraiment en confiance avec son instrument.

\section{Liens entre les pratiques de la composition improvisée et les philosophies de vie}

Les musiciens de l'enquête confirment une réelle interdépendance entre leurs choix de vie personnelle et leur approche artistique. Pour Angelica Sanchez il s'agit d'une destinée :

A mon avis je n'ai pas le choix entre jouer ou ne pas jouer, je crois vraiment que je suis née sur cette Terre pour faire ça. [. . .] Et je m'estime plutôt heureuse que je n'ai pas choisi la musique mais qu'elle m'a choisie. Tu as probablement entendu ça pas mal de fois, mais c'est vraiment ce qu'on ressent, tu vois, comme si c'était le chemin qui t'était tracé ! C'est mon chemin ! Est-ce que j'ai le courage de le suivre? Je n'en sais rien! (rires) ${ }^{20}$

Satoshi Takeishi a rapporté qu'il tombait malade s'il ne se dédiait pas suffisamment à sa propre musique. Todd Capp a remarqué un changement intérieur depuis qu'il a repris la batterie après une dizaine d'année sans en jouer, « Je veux jouer $^{21}$ " alors qu'avant d'arrêter il ressentait, " II fallait que je joue ${ }^{22}$ ". Ils affirment tous le fait qu'ils jouent ce qu'ils sont, et que tout ce qu'ils vivent se retrouve dans leur musique, y compris leurs lectures, les films et les peintures qu'ils regardent et bien sûr la musique qu'ils écoutent. Ils insistent sur leur engagement artistique sans compromis concernant la musique qu'ils créent, ce qui implique un détachement vis à vis du jugement des critiques, du public, d'autres musiciens ainsi que d'eux-mêmes. Comme introduit précédemment, ils s'appliquent à réfuter les pensées parasites d'auto jugement ainsi que la peur du jugement des autres. Pour ne pas se faire prendre par ces pensées, ils utilisent des techniques psychologiques en plus de la pratique de l'instrument. Ces techniques sont explicitées dans le livre Effortless Mastery - Liberating the Master Musician Within du pianiste Kenny Werner (1996) que plusieurs musiciens de l'enquête ont mentionné ; elles se réfèrent aussi à des techniques de méditations, en particulier de zen. Pour Jim Black, l'absence de jugement est directement liée au plaisir qu'il ressent naturellement lorsqu'il joue librement.

Les musiciens ont parfois comparé la pratique de la composition spontanée avec une conversation entre personnes, citons par exemple Mary Halvorson qui décrit la première rencontre avec un autre musicien: " Je pense que c'est seulement une façon de connaître d'autres musiciens et de converser avec eux [. . .] C'est pas tellement d'essayer de comprendre le son de l'autre, mais plutôt de comprendre ton interaction spécifique avec lui. Et tu sais, ça peut vraiment être un moteur et ça peut aussi faire sortir des choses différentes de toi-même. ${ }^{23}$ " Plus tard dans l'entretien, elle garde la même métaphore pour comparer le cas où elle connaît peu l'autre musicien avec le cas où elle le connaît très bien :

C'est presque comme quand tu viens de rencontrer quelqu'un et que t'essayes nerveusement de boucher les trous et de remplir l'espace en parlant, et que les silences ne sont pas forcément aisés. Si bien qu'avec des gens que tu connais, tout est aisé, et du coup tu peux prendre ton temps, et 
laisser les choses se faire, et ne pas hésiter à prendre des risques, tu vois, sans avoir à prouver quoi que ce soit. C'est vraiment agréable et plus tu connais une personne plus tu peux presque te mettre à finir ses phrases, ou anticiper ce qu'elle va dire. ${ }^{24}$

Dans cette citation, nous retrouvons l'importance de limiter les réflexes de jugement entre musiciens pour pouvoir prendre des risques ensemble, ce qui d'après Mary Halvorson est favorisé par le fait de bien connaître les personnes avec qui l'on joue (nous invitons le lecteur à visionner un extrait d'enregistrement de concert où Mary Halvorson improvise avec des musiciens qu'elle connaît de longue date et où la musique paraît être écrite, et pourtant il n'en est rien). Dans le même idée, un musicien explique qu'il faut aussi permettre à la collaboration musicale d'échouer : " II faut vraiment que tu aies la volonté de laisser ces relations ou [. . .] ces communications échouer ou non. ${ }^{25}$ " La corrélation entre les relations sociales et les collaborations musicales ont été discutées par les musiciens de différentes façons. Plusieurs ont mentionné qu'avec le temps, ils n'improvisent plus qu'avec des gens qu'ils apprécient aussi bien artistiquement qu'humainement. Certains séparent les deux êtres, soit le musicien de l'ami, en précisant qu'ils peuvent tout à fait s'entendre musicalement avec des personnes qu'ils trouvent difficiles à vivre, mais que le contraire s'avère plus rare. Daniel Carter ne dissocie pas les personnes de leur expression artistique. II a développé un mode de vie sociale où il cherche à improviser avec toutes les personnes qu'il rencontre, artistes ou non. C'est ainsi que l'auteure s'est retrouvée à jouer avec lui pour pouvoir mener son entretien, échange qui a beaucoup apporté à son enquête car cela lui a permis d'établir des connexions entre l'expérience vécue et les représentations rapportées en entretien.

Cliquez sur l'image pour visionner la video:

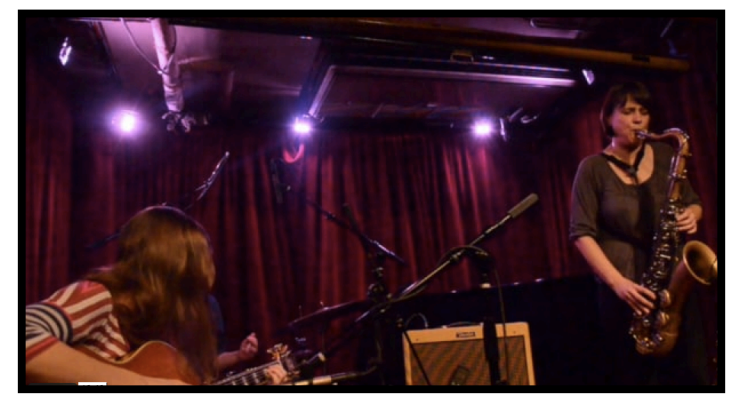

Concert du trio Tom Rainey avec Ingrid Laubrock au saxophone et Mary Halvorson à la guitare au Cornelia Street Cafe, New York, le 30 décembre 2013.

L'approche artistique et le mode de vie de Daniel Carter comportent une dimension politique et sociale très importante. En plus de réfuter les concepts d'élite ou d'expertise, il présente la musique comme un réconfort dans la société : " ouais, tu vois, vu l'état du monde qui nous entoure je me réfugie dans la musique. ${ }^{26}$ " II a aussi mentionné le pouvoir de la composition improvisée face aux dérives de la société, concept également élaboré par Todd Capp : " Si je ne pensais pas qu'il y avait des trucs à apprendre de cette histoire de ne pas être enchainé à la structure, les règles, la routine et les formules, d'être ouvert et libre et spontané sans pour autant se laisser aller, alors je ne ferai pas de musique. ${ }^{27}$ »Plus tard dans l'entretien : « Et de jouer pour moi, c'est de raconter des histoires, et tout est là : je veux prendre les gens par la main et les emmener dans la musique, leur raconter des histoires, pour en sortir avec quelque chose. ${ }^{28}$ "

Todd Capp se réfère au livre Music is My Mistress du pianiste Duke Ellington (1976) pour les liens entre sa philosophie de vie et son approche artistique qui s'inscrit donc pleinement dans le jazz. Les pouvoirs octroyés à cette musique ont aussi été mentionnés par l'un des musiciens de l'enquête : "Des fois d'improviser avec un instrument, ça peut être très apaisant, à d'autres moments, c'est très émotionnel, et puis ça peut aussi être très négatif et dérangeant. $^{29}$ "Plus tard dans l'entretien, ce même musicien rapporte plusieurs expériences de collaboration avec des musiciens en fin de vie, entre autre le pianiste Andrew Hill dont l'énergie revenait lors qu'ils improvisaient. Mary Halvorson a aussi raconté un concert où toutes ses sensations nauséeuses ont disparu le temps de l'improvisation et sont réapparues aussitôt après.

\section{Conclusion}

Cet article rédigé à partir d'entretiens d'improvisateurs professionnels basés à New York situe la pratique de la composition improvisée vis à vis de l'improvisation libre et du free jazz, et il contraste cette pratique avec d'autres contextes stylistiques. Nous avons observé des variations importantes au sein des procédés créateurs de ces musiciens pour improviser librement, allant de l'absence de pratique de l'instrument à une pratique quotidienne exigeante, de l'abstention de penser pendant l'improvisation à une prise de décisions conscientes continuelle. 
Néanmoins, nous avons identifié un consensus important qui concerne l'écoute profonde soit un état d'improvisation nécessaire pour se connecter avec soi-même, avec l'instrument, le public ou les autres musiciens, ou encore avec le tout ou la zone. Ces différents types de connexions, intérieure, extérieure et transcendantale, sont à la source des compositions improvisées tout en étant vécus différemment pour chacun des musiciens et sans influencer leurs choix de collaborations. Les musiciens nous ont aussi fait part de leurs observations concernant les différents pouvoirs que l'improvisation exerçait sur eux-mêmes et/ou sur leur public. Sans qu'aucun n'ait mentionné Libérez votre créativité de Julia Cameron (2007), l'auteure a retrouvé une grande partie de l'approche psychologique de ce livre dans les méthodes des musiciens pour contrer les jugements intérieurs ou extérieurs qui bloquent la créativité et la prise de risques, dans l'écoute des besoins et des désirs de leur enfant intérieur et dans la croyance - partagée seulement par une partie des musiciens de l'enquête - que l'artiste canalise une force créatrice divine. Si des liens ont été constatés entre l'approche artistique et les philosophies de vie sociale, politique et spirituelle des musiciens de l'enquête, ils demeurent strictement individuels et ne rentrent pas non plus en compte dans leurs choix de collaborations. Des personnalités artistiques très différentes improvisent donc ensemble, ce qui permet au public d'apprécier un entrelacement d'approches hétérogènes, entrelacement qui crée l'unicité de cette scène new-yorkaise.

Ce premier tableau ethnographique de cette scène underground prolonge l'enquête menée au sein de la scène jazz américaine des années 1980 par Paul Berliner (1994), d'une analyse phénoménologique et psychologique de l'expérience d'improvisation (Nardonne, 1996) et d'études de cas sur les liens entre improvisation et identité (Macdonald \& Wilson, 2005 ; Sansom, 2007). II complémente aussi des publications récentes de musiciens de la scène de musique improvisée américaine qui ont interrogé leurs collaborateurs, par exemple Conversations du contrebassiste William Parker (2011) et Perpetual Frontier / The Properties of Free Music du guitariste Joe Morris (2012). Sa particularité réside dans la position de l'auteure qui, fascinée par le talent de ces musiciens (ceux de l'enquête et ceux avec qui ils se produisent) en les découvrant sur scène lors d'un premier long séjour à New York en 2010, a commencé par travailler avec eux en tant que réalisatrice d'enregistrements musicaux. Ce rôle professionnel ingrat au sein d'une communauté musicale qui manque de budget et d'intérêt pour l'art de l'enregistrement l'a rapidement amenée à imaginer un projet qui ferait aussi appel à ses aptitudes de chercheure. Cependant, c'est à partir de ses expériences vécues lors d'enregistrements avec cette scène et de la comparaison de ces expériences avec d'autres scènes jazz (Paris et Montréal) et d'autres styles musicaux (la musique classique et contemporaine, l'électroacoustique, le pop-rock) qu'elle a mis en place son projet de recherche et création autour de la pratique de la composition improvisée à New York.

Les entretiens individuels constituent la première étape de ce projet qui inclut des sessions d'écoute guidées à partir d'enregistrements en concert ou en studio, en ensemble ou en solo (enregistrements dont sont issues les vidéos de l'article). Les séances d'écoute suivent des protocoles expérimentaux différents dans le but de recueillir plusieurs perspectives sur cette pratique musicale particulière (Pras, 2015). De plus, l'analyse des données verbales recueillies aussi bien lors des entretiens individuels que des séances d'écoute sont soumis à une analyse collaborative avec les linguistes Caroline Cance et Gilles Cloiseau pour limiter le biais de l'auteure qui vit au sein de cette communauté, et pour identifier les aspects idiosyncratiques et collectifs de cette pratique. En particulier, nous allons effectuer une analyse en profondeur du concept de connexions comme source de création, et de l'utilisation de métaphores par les musiciens pour expliciter leur pratique artistique. Le projet inclut aussi des rencontres improvisées entre les musiciens de l'enquête et d'autres cultures d'improvisation soit le noise (Pras \& Lavergne, 2015) et la musique Hindustani (Pras et al., 2015).

\section{Notes}

1 L'auteure tient à remercier tous les musiciens qui participent à son projet pour leur talent, leur temps et leurs échanges toujours inspirant. Cette enquête a été réalisée grâce à la bourse postdoctorale de l'auteure allouée par le Fonds de Recherche du Québec - Société et Culture (FRQSC).

${ }^{2}$ Les transcriptions ont été revues par le linguiste bilingue Gilles Cloiseau qui s'est aussi occupé des traductions de l'anglais vers le français des citations des musiciens dans cet article.

3 "I don't really think of it in terms of free improvisation versus compositions, versus playing pop tunes, playing standards. [. . .] I just don't really make that distinction, [. . . I mean, the aspiration is to try to create something that is fully realized as a composition, as a symphony, as a string quartet, as anything." (préfère garder l'anonymat)

4 "You're making decisions even though it might be coming from a very honest place on the inside, a very spontaneous place on the inside. You're still making decisions so there is no such thing as free improvisation, it's all spontaneous composition. You're composing things, you're responsible for every sound you make." (Jim Black) 
5 "I think once I started doing that I was like whoa! [. . . ] There is something else also liberating about this and it also helps to inform the other side of me." (préfère garder l'anonymat)

6 "That was such a nice feeling, such a wonderful experience, I mean it can work you know, free jazz doesn't have to be this loud aggressive music that [. . . ] has really given it a very bad reputation." (Todd Capp)

7 "I would meet Daniel Carter in the street and we would talk and he would give me some hope that free jazz was not just a loud aggressive egocentric macho music." (Todd Capp)

8 "If you know of the existence of an idiom called free jazz and if you want to be a part of it, the first thing that you think of is making things up on the spot, and that's with improvisation. Even if you are thinking in terms of bebop or anything else, you think of an improviser as somebody who gets up in front of an audience in real time, develops around space-time in the music." (Matthew Shipp)

9 "In free jazz, if you listen to enough of it they are certain practices, I mean sure you are sitting down and you are making things up, but there is kind of, I don't want to call it clichés but there is ways of going about things that a lot of people that play in the city have figured out pretty fast. So that's not to say specific lines or harmonies or rhythms, but there is kind of ways to corner group activity or solo activity. And if you listen to enough of Sixties' free jazz probably quickly pick up on a few of those things. So that gives you a framework, [. . .] whether it's call and response patterning or like a post-Coltrane way of playing modal, [or] kind of free bopish post-Ornette thing. You know there are a few gestural things that players pick up on pretty closely and [. . .] you're always operating out of some pretext." (Matthew Shipp)

10 "The all idea of playing free is a little bit odd to me because I think there isn't any difference with guys playing bebop or any other music for that matter and playing free. The language might be slightly different, right; it will sound different to your ears. [. . .] Maybe it's not familiar. But the actual music-making part of it, and the information and component you need to make that music are the same. But it took me twenty years to realize that." (Angelica Sanchez)

11 "Sometimes when you play a music that's confined by form, say like a standard or anything that's written over a form, I felt like the big form wasn't addressed enough. Or just the fact that there were arrangements so you had to go to a certain thing [. . .], it wasn't addressing where [. . . I felt the music could also go." (Ingrid Laubrock)

12 "I think if I only improvise I might get stuck in a certain type of rut that I've heard." (Tony Malaby)

13 "I turn the light switch on". (Jim Black)

14 “I feel like I'm receiving dictation" (préfère garder l'anonymat).

15 "Yeah it's musical, it's gesture. Yeah. I'm hearing it and [. . .] I'm so there, I'm so present and so listening [. . .] and so aware of it, and I'm so connected to the other. [. . .] If I'm by myself I am so connected to the audience and the space and the instrument. If I'm playing with other people we are also connected [. . .], which doesn't mean that we are all hearing the same thing but we're all so connected that the piece unfolds by itself. I'm just getting out of the way of it, and that's the best feeling and to me that's the one where it's really the freest improvisation. Because it's not been conditioned by any willfulness that's outside of the piece. This is the first time that piece has ever been played." (préfère garder l'anonymat).

16 "What I'm trying to do when I improvise, when I sit down to play by myself or with other people, is to not think. The goal is to get yourself out of the way, and just channel whatever is going to come out." (Angelica Sanchez)

17 "I don't know what it means to think, you're trying to really have all of this be instinct and you try to take in information but instantly react and within the instantaneousness of the reaction it's not really thinking, that's reacting." (Matthew Shipp)

18 "One part of you is kind of like a mixer, you have all these different knobs. So one of you is playing and is completely like just playing you know, thinking, dreaming, [and] letting it go. And the other one has a knob for [. . .] brain activity or gestural, physical, like nervous system, heart, memory." (préfère garder l'anonymat) 
19 "The feeling is so primitive in how creation is taking part and it's so intuitive and basic [. . .] At the same time that's going on, there is very evolved strategies and thinking and being compositional and making very intelligent types of tightrope choreography, and having the balance of both things. And that to me having both those things really spinning in a fast frequency, I don't get from playing other types of music." (Tony Malaby)

20 "In my mind there isn't any choice to play or not play, like I really feel that I was born on earth to do this! [. . .] And I feel lucky enough [that] I didn't choose music and music chose me. You probably heard that a bunch, but that's how it really feels like, you know like this is your path! This is my path! Do I have the courage to walk it? I don't know! ((Laughs))." (Angelica Sanchez)

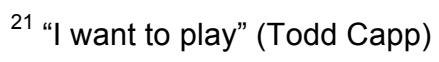

23 "I think it's just a way to get to know other musicians and have a conversation. [. . . It's less about getting to know how someone sounds and more [about] understanding your specific interaction with somebody. And you know [it] can really push you and you know it can bring out different things in yourself too." (Mary Halvorson)

24 "It's almost like if you just met someone and you're just kind of nervously filling space by talking and silence is maybe not comfortable. But with people you know, anything is comfortable so you can really take your time and let stuff happen and feel comfortable to take risks, you know without trying to prove anything. That's really nice and the more you get to know someone you can almost start to finish their sentences or anticipate where they are going, which can be nice as well." (Mary Halvorson)

25 "you really have to [. . .] be willing to allow that relationship or [. . .] those communications to fail or not." (préfère garder l'anonymat)

26 "yeah man the way the world is I am taking refuge in music." (Daniel Carter)

27 "If I didn't think there were some things that somebody could learn from this about not being bound by structure and rules and routine and formula, being open and being free and being spontaneous but not being self-indulgent, then $I$ wouldn't play." (Todd Capp)

28 "And playing music to me is about telling stories, and life is about telling stories, and that's the thing: I want to take people along in the music and tell stories, and you come out with something." (Todd Capp)

29 "sometimes improvising on the instrument can be very healing, other times it can be very emotional and other times it can be very negative and disruptive." (préfère garder l'anonymat)

\section{Références bibliographiques}

Bailey, Derek. Improvisation: Its Nature And Practice In Music. Boston : Da Capo, 1993. Imprimé.

Berliner, Paul F. Thinking in Jazz: The Infinite Art of Improvisation. Chicago: U of Chicago P, 1994. Imprimé.

Borgo, David. "Negotiating Freedom: Values and Practices in Contemporary Improvised Music." Black Music Research Journal 22.2 (2002): 265-188. Imprimé.

Cameron, Julia. Libérez votre créativité. Paris: J'ai lu - Flammarion, 2007. Imprimé. Des détails de la version originale en anglais The Artist's Way (2002) sont disponibles sur http://juliacameronlive.com/the-artists-way/

Canonne, Clément. "Improvisation collective libre et processus de création musicale: création et créativité au prisme de la coordination." Revue de musicologie 98.2 (2012): np. Imprimé.

Carles, Philippe, et Jean-Louis Comolli. Free Jazz / Black Power. Paris: Folio Gallimard, 2000. Imprimé. 
Corbin, Juliet, et Anselm L. Strauss. Basics of Qualitative Research: Techniques and procedures for developing grounded theory. Thousand Oaks: Sage, 2008. Imprimé.

Delepaut, Gaëlle. "Questionner: Comment? Pourquoi? le choc des questions, le poids des mots." Le sentir et le dire. Ed. D. Dubois. Paris: L'Harmattan, 2009. 157-188. Imprimé.

Drott, Eric. Music and May 1968 in France: Practices, roles, representations. New York: Cambridge U P, 2011. Imprimé.

Ellington, Duke. Music is My Mistress. Boston: Da Capo, 1976. Imprimé.

Jones, LeRoi [Amiri Baraka]. Le people du blues: La musique noire dans l'Amérique blanche. Paris: Folio Gallimard, 1997. Imprimé.

Lewis, George E. "Improvised music after 1950: Afrological and Eurological perspectives." Black Music Research Journal 16.1 (1996): 91-122. Imprimé.

McCormack, Ryan Sawyer. "Outside of the Self: Subjectivity, the Allure of Transcendence, and Jazz Historiography." Critical Studies in Improvisation / Études critiques en improvisation 8.1 (2012). Web.

Macdonald, Raymon, et Graeme Wilson. "Musical identities of professional jazz musicians: a focus group investigation." Psychology of Music 33.4 (2005): 395-417. Imprimé.

Morris, Joe. Perpetual Frontier / The Properties of Free Music. New York: Riti, 2012. Imprimé.

Nardone, Patricia L. "The experience of improvisation in music: a phenomenological, psychological analysis." Diss. Saybrook Institute, 1996. Imprimé.

Parker, William. Conversations. Paris: RogueArt, 2012. Imprimé.

Pras, Amandine, et Grégoire Lavergne. "L'échantillonnage dans l'improvisation : Rencontre de deux instigateurs du free jazz avec un jeune artiste de la scène noise à New York." Musicae Scientiae 22.4 (2015): 433-451. Imprimé.

Pras, Amandine, Caroline Cance, et Gilles Cloiseau. "Rencontres improvisées entre New York et Kolkata : Analyse collaborative d'une étude transculturelle." Présenté au colloque international Analyser les processus de création musicale (TCPM). Paris. 9 Oct. 2015. Communication.

Pras, Amandine. "Research \& creative adventures with distinguished improvisers from New York City". vs. Interpretation. Ed. D. Rothenberg. Prague : Agosto, 2015. 119-129. Imprimé.

Roueff, Olivier. Jazz, les échelles du plaisir. Paris: La Dispute, 2013. Imprimé.

Sansom, Matthew James. "Improvisation \& Identity: a Qualitative Study." Critical Studies in Improvisation / Études critiques en improvisation 3.1 (2007). Web.

Werner, Kenny. Effortless Mastery - Liberating the Master Musician Within. New Albany: Jamey Aebersold Jazz, 1996. Web. Version pdf disponible sur: http://kennywerner.com/effortless-mastery

\section{Références discographiques}

Coltrane, John. Live ! at the Village Vanguard. Produit par Bob Thiele. Santa Monica: Impulse!, 1962.

---. Meditations. Produit par Bob Thiele. Santa Monica: Impulse!, 1966.

Quintet, Miles Davis. Miles Smiles. Produit par Teo Macero. New York: Columbia, 1967. 
Ornette Coleman Double Quartet. Free Jazz - A collective improvisation. Produit par Nesuhi Ertegün. New York: Atlantic, 1961.

Shepp, Archie. Fire Music. Produit par Bob Thiele. Santa Monica: Impulse!, 1965. 